\title{
IRTS observation of the mid-infrared spectrum of the zodiacal emission
}

\author{
T. Ootsubo ${ }^{1}$, T. Onaka ${ }^{1}$, I. Yamamura ${ }^{1, *}$, T. Tanabé ${ }^{2}$, T. L. Roellig ${ }^{3}$, K.-W. Chan ${ }^{3}$, and T. Matsumoto ${ }^{4}$ \\ ${ }^{1}$ Department of Astronomy, School of Science, University of Tokyo, Bunkyo-ku, Tokyo 113-0033, Japan \\ 2 Institute of Astronomy, Faculty of Science, University of Tokyo, Osawa, Tokyo 181-8588, Japan \\ ${ }^{3}$ NASA Ames Research Center, MS 245-6, Moffett Field, CA 94035-1000, U.S.A. \\ ${ }^{4}$ Institute of Space and Astronautical Science, Sagamihara, Kanagawa 229-8510, Japan
}

(Received October 8, 1997; Revised February 6, 1998; Accepted February 21, 1998)

\begin{abstract}
We present the mid-infrared spectrum $(3-12 \mu \mathrm{m})$ of the zodiacal emission obtained by the Infrared Telescope in Space (IRTS), the first Japanese cryogenically cooled orbital infrared telescope. The Near-Infrared Spectrometer (NIRS) on board IRTS provided the spectrum of 3-4 $\mu \mathrm{m}$, while that of 4.5-11.7 $\mu \mathrm{m}$ has been observed by the Mid-Infrared Spectrometer (MIRS). In this paper we present the data reduction and results of the observations by MIRS. Spectra of the background emission at high galactic latitudes $\left(|b|>30^{\circ}\right)$ have been extracted from the MIRS observations by excluding point sources. The observed sky brightness has a clear dependence on the ecliptic latitude, indicating that the zodiacal emission dominates in the mid-infrared sky brightness. On the other hand, the spectral shape does not show any appreciable dependence on the ecliptic latitude for $\beta=0^{\circ}-75^{\circ}$. The spectrum combining the NIRS and MIRS observations can be fitted by a grey body radiation at $250 \mathrm{~K}$, but excess emission is seen in the 3-6 $\mu \mathrm{m}$ range. Alternatively, the spectrum of the zodiacal emission can be reproduced fairly well by a grey body at $280 \mathrm{~K}$ with an excess around $10 \mu \mathrm{m}$. In this case the excess may be attributed to a silicate emission band. Other than these excesses, no spectral features above the $10 \%$ level are seen in the MIRS spectrum.
\end{abstract}

\section{Introduction}

The zodiacal emission is the thermal emission from interplanetary dust particles (IDPs) and composes the dominant background radiation in the mid-infrared wavelengths. Mid-infrared observations of the zodiacal emission provide important information on IDPs and complements studies of the zodiacal light in the visual range. The Zodiacal Infrared Project (ZIP), the Infrared Astronomical Satellite (IRAS), and the Diffuse Infrared Background Experiment (DIRBE) on the Cosmic Background Explorer (COBE) missions surveyed wide areas of the sky in the infrared and observed the zodiacal emission with broad-band photometry (Hauser and Houk, 1984; Murdock and Price, 1985; Reach et al., 1996a), enabling detailed modelings of the spatial distribution and/or optical properties of the IDP (e.g., Hong and Um, 1987; Dumont and Levasseur-Regourd, 1988; Reach, 1988, 1992; Giese and Kneissel, 1989; Reach et al., 1996a). Spectroscopic studies, on the other hand, are expected to provide significant information on the compositions and size distribution of IDPs.

Recently, spectroscopic observations of the zodiacal emission near the ecliptic plane have been made by the camera on the Infrared Space Observatory (ISOCAM) in the midinfrared region (5-16 $\mu \mathrm{m})$ (Reach et al., 1996b). Reach (1997) suggests that the ISOCAM spectrum can be fitted by grey body radiation at $270 \mathrm{~K}$ with a broad excess emission feature in the 9-11 $\mu \mathrm{m}$ range.

*Also at SRON, Groningen, 9700 AV Groningen, The Netherlands.

Copy right (c) The Society of Geomagnetism and Earth, Planetary and Space Sciences (SGEPSS); The Seismological Society of Japan; The Volcanological Society of Japan; The Geodetic Society of Japan; The Japanese Society for Planetary Sciences.
The near-infrared spectrum of the zodiacal light and emission in the spectral range of $1.4-4 \mu \mathrm{m}$ has been obtained by the Near-Infrared Spectrometer (NIRS) on board the Infrared Telescope in Space (IRTS) (Matsumoto et al., 1996). They found that the spectrum shorter than $3.2 \mu \mathrm{m}$ is dominated by scattered solar radiation and is well fitted by a grey body of $6000 \mathrm{~K}$. On the other hand, the spectrum at longer wavelengths shows a clear excess relative to the scattered component, indicating that the thermal emission from IDPs dominates for $\lambda>3.2 \mu \mathrm{m}$.

This paper presents the results of the mid-infrared observations $(4.5-11.7 \mu \mathrm{m})$ of the zodiacal emission obtained by the Mid-Infrared Spectrometer (MIRS) also on board IRTS (Roellig et al., 1994). Combining these results with the NIRS spectrum, the spectrum of the zodiacal emission for the spectral range of 3-12 $\mu \mathrm{m}$ is obtained around the ecliptic plane and its characteristics are discussed.

\section{Observations}

IRTS was the first Japanese cryogenically cooled orbital infrared telescope and surveyed about $7 \%$ of the entire sky (Murakami et al., 1996). MIRS was one of the four focal plane instruments on board IRTS. It was a low-resolution spectrometer with a field of view of $8^{\prime} \times 8^{\prime}$, and covered the spectral range of 4.5-11.7 $\mu \mathrm{m}$ with 32 discrete detectors. The spectral resolution was approximately $0.3 \mu \mathrm{m}$ and was optimized for the spectroscopic study of diffuse extended emission (Roellig et al., 1994). The flight calibration and performance of MIRS are given in Roellig et al. (1996) and Tanabé et al. (1997). The procedures of correction of the cosmic ray hits and point source extraction are given in Yamamura et al. (1997). In the followings we describe the data 


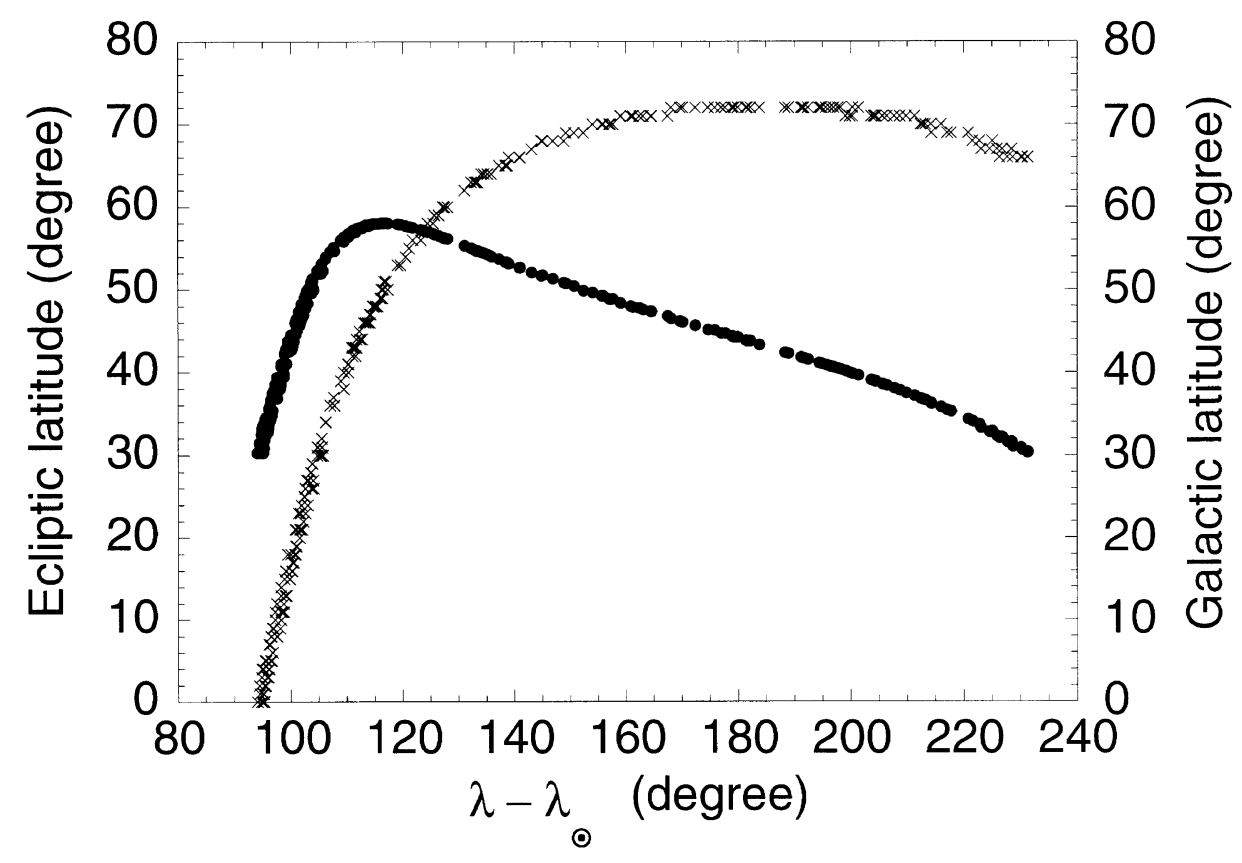

Fig. 1. Selected sky areas used for the analysis are shown in the ecliptic coordinates $(\lambda-\lambda \odot, \beta)$ by crosses (left ordinate). Their galactic latitudes are shown by filled circles (right ordinate).

reduction and results of the zodiacal emission observations by MIRS. The data reduction and results of the NIRS observations have been given in Matsumoto et al. (1996).

The IRTS observations were made from 1995 March 29 to April 26. In this paper we present the data of two days, April 19 and April 20, where the performance of the instrument was the most stable and disturbances, such as those due to satellite maneuvers, were minimal. We selected sky area with high galactic latitudes $\left(|b|>30^{\circ}\right)$ in order to minimize possible contributions from faint stars in the Galactic disk. We smoothed the raw data over time periods of $5 \mathrm{~s}$, in regions where no point sources were seen. We finally had about 500 data sets to be used for the present analysis. Dark currents were subtracted and the raw signal converted into surface brightnesses based on flight absolute calibrations and beam profile measurements (cf., Onaka et al., 1996).

In Fig. 1, we show the selected sky areas in the ecliptic coordinates. Here, $\lambda$ and $\beta$ represent the ecliptic longitude and latitude of the selected regions, respectively, while $\lambda_{\odot}$ represents the ecliptic longitude of the Sun. Their galactic latitudes are shown in the right vertical axis. The scan paths crossed the ecliptic plane at $\lambda-\lambda_{\odot} \sim 90^{\circ}$, and reached $\beta \sim 75^{\circ}$, while the galactic latitude changed from $30^{\circ}$ to $60^{\circ}$. The sky area where the data were sampled is rather limited due to the one-month observation period of IRTS, but it is still sufficiently extensive to investigate the zodiacal emission.

\section{Data Reduction and Results}

We averaged the data sets in $5^{\circ}$ intervals of the ecliptic latitude along the scan and obtained the mean sky brightness. In Fig. 2, the ecliptic dependence of the surface brightness is shown for four typical wavelength bands in the April 20 data. The observed sky brightness shows a clear dependence on the ecliptic latitude. Furthermore, it can be shown that all the wavelength channels of MIRS have a similar dependence on the latitude, indicating that the spectral shape does not change appreciably with the ecliptic latitude. The spectral shapes of two typical regions $\left(12 .^{\circ} 5<\beta<20 .^{\circ} 0,71 .^{\circ} 3<\right.$ $\left.\beta<73 .^{\circ} 0\right)$ are quite similar to each other as shown in Fig. 3. The sky brightnesses at the $4.9 \mu \mathrm{m}$ and $12 \mu \mathrm{m}$ bands of the DIRBE observations have been obtained from the weekly skymaps by averaging the data over the same areas and solar elongations as those of the MIRS spectra and they are shown also in Fig. 3 by squares. The DIRBE data points are colorcorrected by a grey body of $250 \mathrm{~K}$. Taking account of the calibration uncertainty of the MIRS (Tanabé et al., 1997), the MIRS spectra are in agreement with the DIRBE data.

The observed mid-infrared sky brightness consists of the potentially uncorrected instrumental dark current, if any, the zodiacal emission, the integrated light of the galactic components (faint stars and emission from the interstellar medium), and the extragalactic emission. The spectrum of the zodiacal emission has been obtained by subtracting the background emission at high ecliptic latitudes $\left(71 .^{\circ} 3<\beta<73 .^{\circ} 0\right)$ from that at low ecliptic latitudes $\left(12 .^{\circ} 5<\beta<20 .^{\circ} 0\right)$. Both data sets were chosen to have the same galactic latitudes $\left(40^{\circ}<b<45^{\circ}\right)$ in order to cancel out the contribution from the remaining dark current, the faint stellar contribution, and the extragalactic emission. No apparent dependence of the spectral shape on the ecliptic latitude ensures that this procedure will not introduce any spurious features in the resulting spectrum.

The interstellar medium has been shown to have distinct band features, so-called "unidentified infrared bands", in the mid-infrared range (Mattila et al., 1996; Onaka et al., 1996, 1997). Dwek et al. (1997) indicates that the contribution of this component is several $\times 10^{-6} \mathrm{~W} \mathrm{~m}^{-2} \mathrm{sr}^{-1}$ per $10^{20} \mathrm{HI}$ 


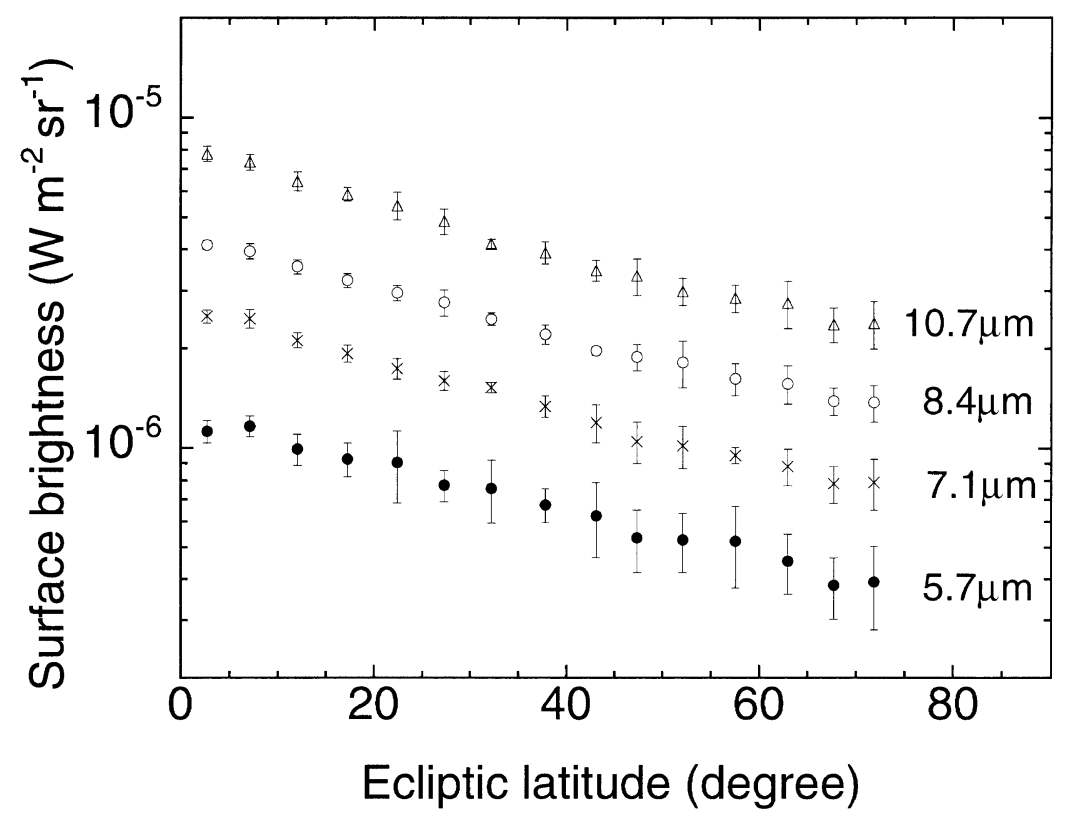

Fig. 2. The mean sky brightnesses of the April 20 of four wavelength bands at $5.7 \mu \mathrm{m}$ (filled circles), $7.1 \mu \mathrm{m}$ (crosses), $8.4 \mu \mathrm{m}$ (open circles), and $10.7 \mu \mathrm{m}$ (open triangles) against the ecliptic latitude.

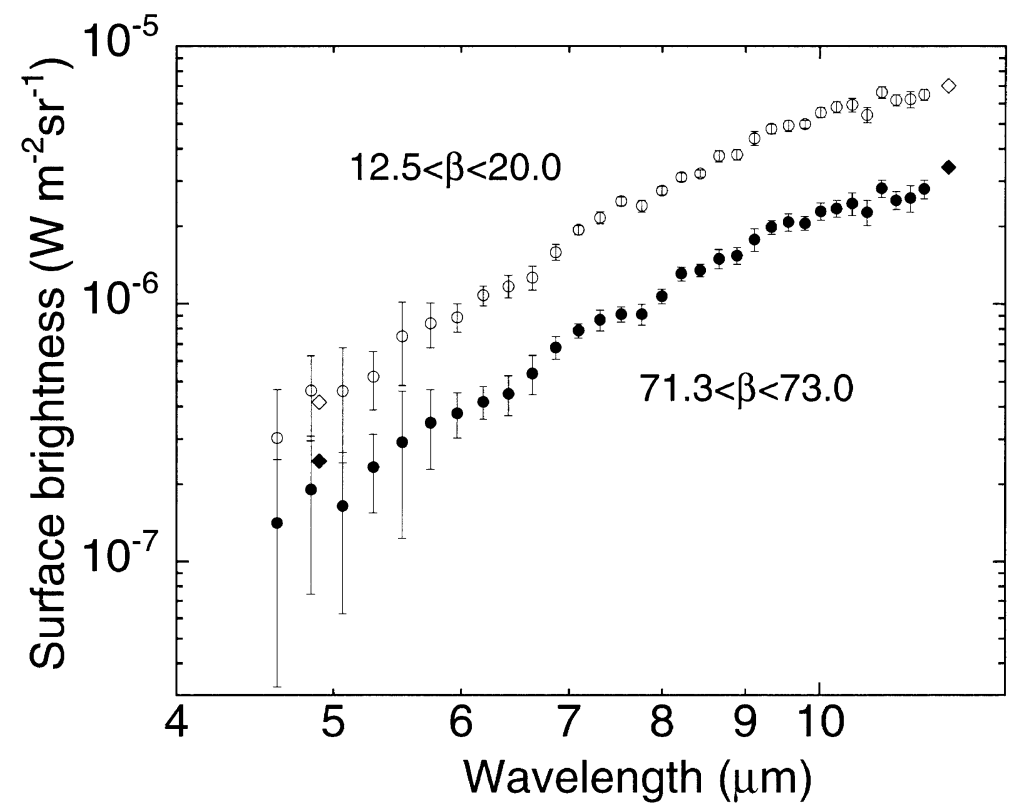

Fig. 3. MIRS spectra of the sky brightness. The spectra at the low ecliptic latitudes $\left(12 .{ }^{\circ} 5<\beta<20 .{ }^{\circ} 0\right)$ and the high ecliptic latitudes $\left(71 .^{\circ} 3<\beta<73 .{ }^{\circ} 0\right)$ are plotted by open and filled circles, respectively. Both data sets have the same galactic latitudes $\left(40^{\circ}<b<45^{\circ}\right)$. The DIRBE brightnesses of the same celestial regions with the same solar elongations at the $4.9 \mu \mathrm{m}$ and $12 \mu \mathrm{m}$ bands are shown by squares. The DIRBE data have been color-corrected by a grey body of $250 \mathrm{~K}$.

atoms $\mathrm{cm}^{-2}$ at the band peaks. The HI column density of the observed direction of the sky is typically several $\times 10^{20}$ $\mathrm{cm}^{-2}$ and thus this component could still be observable in the subtracted spectrum. Further observational studies are needed to make better estimate of the contribution of this component. The spectrum of the difference between two regions obtained here, however, does not indicate any UIR band features and we assume that the contribution from the interstellar medium is negligible in this study.

The zodiacal emission spectrum, thus obtained, was scaled to the observed $7.09 \mu \mathrm{m}$ brightness of the region of $|b|<10^{\circ}$. We show this scaled MIRS spectrum of the zodiacal emission in Fig. 4. In Fig. 4, we also show the spectrum of the zodiacal light of $1.4-4.0 \mu \mathrm{m}$, which was obtained by NIRS for the same sky area as used in this study (Matsumoto et al., 1996). It can be seen that the MIRS spectrum is connected smoothly 


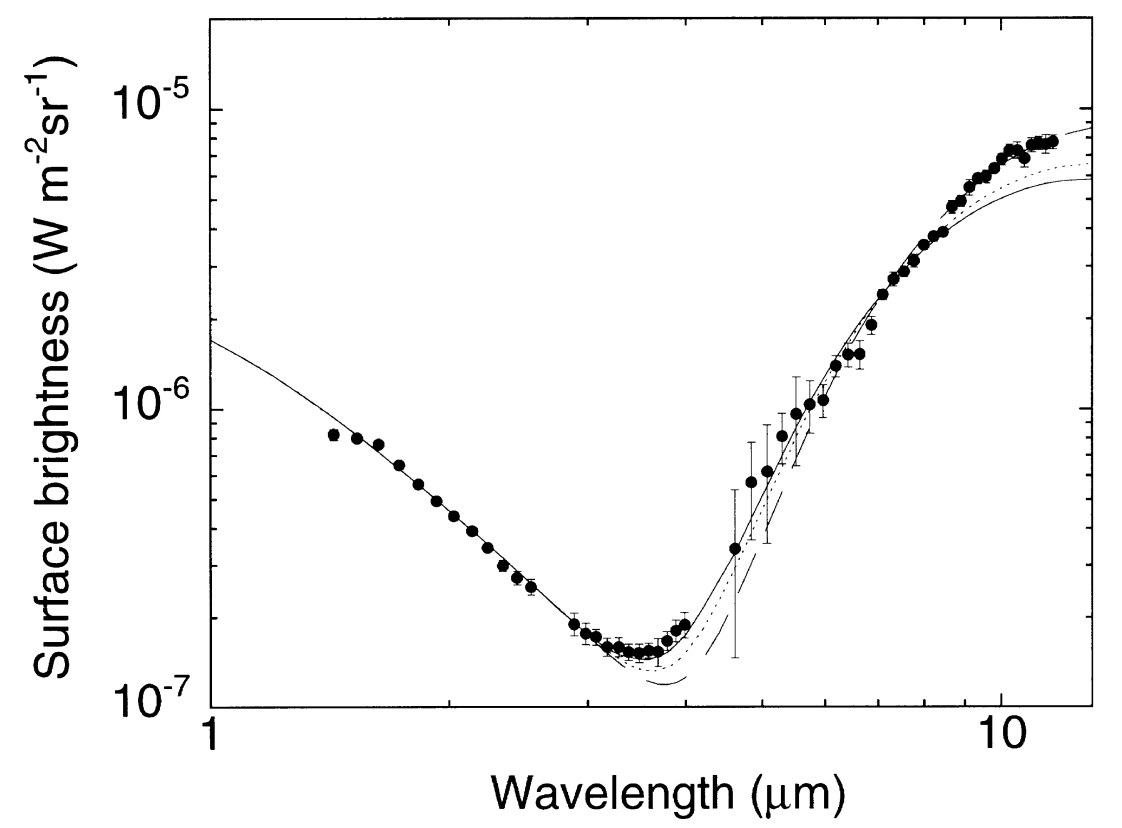

Fig. 4. The NIRS and MIRS spectra of the zodiacal light and emission. The MIRS spectrum was scaled to the $7.09 \mu \mathrm{m}$ brightness of the region of $|\beta|<10^{\circ}$. The NIRS spectrum was scaled to the $1.83 \mu \mathrm{m}$ brightness (Matsumoto et al., 1996). The dashed line indicates the sum of the spectra of $6000 \mathrm{~K}$ grey body scaled to the observed $1.83 \mu \mathrm{m}$ brightness and that of $250 \mathrm{~K}$ grey body scaled to the observed $7.09 \mu \mathrm{m}$ brightness. The solid line indicates the sum of $6000 \mathrm{~K}$ and $280 \mathrm{~K}$, and the dotted line indicates the sum of $6000 \mathrm{~K}$ and $270 \mathrm{~K}$ grey bodies similarly scaled.

with the NIRS spectrum. The short wavelength part of the NIRS spectrum $(\lambda<3.2 \mu \mathrm{m})$ is attributed to the scattered light of the solar radiation by IDPs and can be fitted well by a grey body of $6000 \mathrm{~K}$.

\section{Discussion}

Dumont and Levasseur-Regourd (1988) suggested from the analysis of the wide-band photometry data of ZIP and IRAS that the temperature of the zodiacal emission inferred from each data set is different, being $298 \mathrm{~K}$ and $257 \mathrm{~K}$, respectively, when the dust is assumed to emit as a grey body. The difference may be attributed to the difference in their absolute calibrations. The latest spectroscopic study by ISOCAM indicates that the mid-infrared spectrum $(5-16 \mu \mathrm{m})$ can be fitted by a grey body at $270 \mathrm{~K}$ (Reach, 1997) and suggests a broad emission hump of $10 \%$ in the $9-11 \mu \mathrm{m}$ region.

As shown in Fig. 4, the MIRS spectrum of the zodiacal emission $(6-12 \mu \mathrm{m})$ can be fitted fairly well by a grey body curve at $250 \mathrm{~K}$ (dashed line). In this fit, however, excess emission appears in the range from $3 \mu \mathrm{m}$ of the NIRS spectrum to $6 \mu \mathrm{m}$ of the MIRS spectrum. The MIRS spectrum is smoothly connected to the NIRS spectrum, and thus, the excess in this fit is rather secure. On the other hand, the spectrum from $3 \mu \mathrm{m}$ to $8 \mu \mathrm{m}$ matches well with a $280 \mathrm{~K}$ grey body (solid line), which is about the radiative equilibrium temperature of a grey body at the earth's orbit. In this case, excess emission is seen at wavelengths longer than $9 \mu \mathrm{m}$, as suggested by Reach (1997) in the ISOCAM spectrum. A slight depression is also seen in the range 5.5-7 $\mu \mathrm{m}$. The 9$12 \mu \mathrm{m}$ excess seen is larger $(\geq 20 \%)$ than that indicated by Reach (1997), who fits the spectrum with that of a grey body at temperature of $270 \mathrm{~K}$. A fit of the IRTS spectrum with a $270 \mathrm{~K}$ grey body results in a less excess in the $10 \mu \mathrm{m}$ region, but shows a slight excess at 3-4 $\mu \mathrm{m}$ of the NIRS spectrum. The derived temperature thus depends on the spectral range where the fit is attempted and it is difficult to put a decisive conclusion only from the present data set. The shape of the MIRS spectrum is in agreement with the ISOCAM spectrum as a whole. The absolute brightness cannot be directly compared because the MIRS did not observe the position of the ISOCAM observations with the same solar elongation.

The temperature of IDPs is sensitive to the size and composition of the particles. In addition, if the presence of the excess emission feature is real, it should provide significant information on the nature of IDPs. The size distribution of the interplanetary meteoroids (Grün et al., 1985) indicates that the zodiacal light is dominated by large particles with radii of around $30 \mu \mathrm{m}$, which have temperatures around $280 \mathrm{~K}$. The excess at 3-6 $\mu \mathrm{m}$, if real, could be band emission of the particles or particles with high temperatures $(\geq 400 \mathrm{~K})$. Such high temperatures may be obtained if the particles are smaller than the submicron size and/or have high absorptivity (Reach, 1988).

Matsumoto et al. (1996) suggest from the near-infrared zodiacal light observations by NIRS that IDPs may consist of the material similar to the S-type asteroids. If IDPs are dominated by silicate-type particles of the size distribution with an enhancement of submicron particles, such as the lunar microcrater distribution (Grün et al., 1985), they may show band features in the $10 \mu \mathrm{m}$ region (Reach et al., 1996b). A recent study of asteroids also suggests the presence of band features in the $10 \mu \mathrm{m}$ region of the spectrum of Ceres (Cohen et al., 1998). Therefore, the presence of features in the $10 \mu \mathrm{m}$ region of the zodiacal emission is not unexpected and the near-infrared zodiacal light and the mid-infrared zodiacal emission may be interpreted consistently in terms of 
the silicate-type IDPs. These silicate particles must be absorptive enough to get to high temperatures. "Astronomical silicate" (Draine and Lee, 1984) is a likely candidate since it has large absorptivity relative to terrestrial silicates. However, it still does not provide a reasonable fit to the observed hump (Reach et al., 1996b). The present investigation clearly indicates the significance of spectroscopic study in determining the nature of IDPs and further investigations are certainly warranted.

Other than these possible broad features we do not see any other features at more than the $10 \%$ level in the MIRS spectrum of the zodiacal emission. Several small features may be seen, but they are within calibration errors.

Acknowledgments. We would like to thank all the members of the IRTS team for their kind support and helpful discussions, the members of the NIRS team for their help in preparing the NIRS data, and Martin Cohen for providing us the latest Ceres data prior to the publication. I. Y. is supported by the JSPS Research Fellowships for Young Scientists. T. O. and T. T. are supported in part by a Grant-in-Aid from the Ministry of Education, Science, Sports and Culture in Japan. The IRTS is a joint mission between ISAS and NASA. We are grateful to IPAC for their help in the pointing reconstruction of the IRTS. The COBE data sets were developed by the NASA Goddard Space Flight Center under the guidance of the COBE Science Working Group and were provided by the NSSDC.

\section{References}

Cohen, M., F. C. Witteborn, T. Roush, J. Bregman, and D. Wooden, Spectral irradiance calibration in the infrared. VIII. 5-14 $\mu \mathrm{m}$ spectroscopy of the asteroids, Ceres, Vesta, and Pallas, Astron. J., 115, 1671-1679, 1998.

Draine, B. T. and H. M. Lee, Optical properties of interstellar graphite and silicate grains, Astrophys. J., 285, 89-108, 1984.

Dumont, R. and A.-C. Levasseur-Regourd, Properties of interplanetary dust from infrared and optical observations, Astron. Astrophys., 191, 154-160, 1988.

Dwek, E., R. G. Arendt, D. J. Fixsen, T. J. Sodroski, N. Odegard, J. L. Weiland, W. T. Reach, M. G. Hauser, T. Kelsall, S. H. Moseley, R. F. Silverberg, R. A. Shafer, J. Ballester, D. Bazell, and R.Isaacman, Detection and characterization of cold intersteller dust and polycyclic aromatic hydrocarbon emission, from $C O B E$ observations, Astrophys. J., 475, 565-579, 1997.

Giese, R. H. and B. Kneissel, Three-dimensional models of the zodiacal dust cloud, Icarus, 81, 369-378, 1989.

Grün, E., H. A. Zook, H. Fechtig, and R. H. Giese, Collisional balance of the meteoritic complex, Icarus, 62, 244-272, 1985.

Hauser, M. G. and J. R. Houk, The zodiacal background in the IRAS data, in Proceedings of Light on Dark Matter, edited by F. P. Israel, pp. 39-44, Reidel, Dordrecht, 1984.

Hong, S. S. and I. K. Um, Inversion of the zodiacal infrared brightness integral, Astrophys. J., 320, 928-935, 1987.

Matsumoto, T., M. Kawada, H. Murakami, M. Noda, S. Matsuura, M. Tanaka, and K. Narita, IRTS observation of the near-infrared spectrum of the zodiacal light, Publ. Astron. Soc. Japan, 48, L47-L51, 1996.

Mattila, K., D. Lemke, L. K. Haikala, R. J. Laureijs, A. Léger, K. Lehtinen, Ch. Leinert, and P. G. Mezger, Spectrophotometry of UIR bands in the diffuse emission of the galactic disk, Astron. Astrophys., 315, L353-L356, 1996.

Murakami, H., M. M. Freund, K. Ganga, H. Guo, T. Hirao, N. Hiromoto, M. Kawada, A. E. Lange, S. Makiuti, H. Matsuhara, T. Matsumoto, S. Matsuura, M. Murakami, T. Nakagawa, M. Narita, M. Noda, H. Okuda, K. Okumura, T. Onaka, T. L. Roellig, S. Sato, H. Shibai, B. J. Smith, T. Tanabé, M. Tanaka, T. Watabe, I. Yamamura, and L. Yuen, The IRTS (Infrared Telescope in Space) mission, Publ. Astron. Soc. Japan, 48, L41-L46, 1996.

Murdock, T. L. and S. D. Price, Infrared measurements of zodiacal light, Astron. J., 90, 375-386, 1985.

Onaka, T., I. Yamamura, T. Tanabé, T. L. Roellig, and L. Yuen, Detection of the mid-infrared unidentified bands in the diffuse galactic emission by IRTS, Publ. Astron. Soc. Japan, 48, L59-L63, 1996.

Onaka, T. and the IRTS team, IRTS observations of the unidentified infrared bands in the diffuse galactic light, in Proceedings of the Diffuse Infrared Radiation and the IRTS, ASP Conference Series Vol. 124, edited by H. Okuda, T. Matsumoto, and T. L. Roellig, pp. 170-177, 1997.

Reach, W. T., Zodiacal emission I. Dust near the earth's orbit, Astrophys. J., 335, 468-485, 1988.

Reach, W. T., Zodiacal emission III. Dust near the asteroid belt, Astrophys. J., 392, 289-299, 1992.

Reach, W. T., The structured zodiacal light: IRAS, COBE, and ISO observations, in Proceedings of the Diffuse Infrared Radiation and the IRTS, ASP Conference Series Vol. 124, edited by H. Okuda, T. Matsumoto, and T. L. Roellig, pp. 33-40, 1997.

Reach, W. T., B. A. Franz, T. Kelsall, and J. L. Weiland, DIRBE observations of the zodiacal light, in Proceedings of Unveiling the Cosmic Infrared Background, AIP Conference Series Vol. 348, edited by E. Dwek, pp. 3746, College Park, MD, 1996a.

Reach, W. T., A. Abergel, F. Boulanger, F.-X. Désert, M. Perault, J.-P. Bernard, J. Blommaert, C. Cesarsky, D. Cesarsky, L. Metcalfe, J.-L. Puget, F. Sibille, and L. Vigroux, Mid-Infrared spectrum of the zodiacal light, Astron. Astrophys., 315, L381-L384, 1996b.

Roellig, T. L., T. Onaka, T. J. McMahon, and T. Tanabé, The Mid-Infrared Spectrometer on the Infrared Telescope in Space (IRTS) mission, Astrophys. J., 428, 370-376, 1994.

Roellig, T. L., K. Mochizuki, T. Onaka, T. Tanabé, I. Yamamura, and L. Yuen, Flight Performance of the Mid-Infrared Spectrometer on the Infrared Telescope in Space mission, Proc. SPIE, 2817, 258-266, 1996.

Tanabé, T., I. Yamamura, T.Onaka, K.-W. Chan, T. L. Roellig, and M. Cohen, The absolute sensitivity calibration of the IRTS/MIRS, in Proceedings of the Diffuse Infrared Radiation and the IRTS, ASP Conference Series Vol. 124, edited by H. Okuda, T. Matsumoto, and T. L. Roellig, pp. 25-30, 1997.

Yamamura, I. and the IRTS team, Point source observations by the IRTS, in Proceedings of the Diffuse Infrared Radiation and the IRTS, ASP Conference Series Vol. 124, edited by H. Okuda, T. Matsumoto, and T. L. Roellig, pp. 72-81, 1997.

T. Ootsubo (e-mail: ootsubo@astron.s.u-tokyo.ac.jp), T. Onaka, I. Yamamura, T. Tanabé, T. L. Roellig, K.-W. Chan, and T. Matsumoto 